\title{
The Prevention and Control Experience of Maternal Health Care from Chengu, China During the COVID-19 Epidemic
}

This article was published in the following Dove Press journal: Risk Management and Healthcare Policy

\section{Chunrong Li* \\ Li Tang* \\ Yingjuan Luo \\ Ting Lai \\ Lingling $\mathrm{Gu}$ \\ Yumei Wang \\ Liu Yang \\ Longrong Ran \\ Rongkang Wen \\ Yonghong Lin}

Chengdu Women's and Children's Central Hospital, School of Medicine, University of Electronic Science and Technology of China, Chengdu, Sichuan, People's Republic of China

*These authors contributed equally to this work
Correspondence: Yonghong Lin Chengdu Women's and Children's Central Hospital, School of Medicine, University of Electronic Science and Technology of China, No. 1617, Riyue Avenue, Qingyang District, Chengdu 61009I, People's

Republic of China

Tel +86 I380803I895

Fax +862861866003

Emailyhlin1022@I63.com

\begin{abstract}
Four months after the first case of COVID-19 was diagnosed in Wuhan, the national epidemic has been effectively controlled in China. In Chengdu, the capital city of Sichuan Province, several management measures, which have been proven to be effective, are taken to prevent pregnant women from being infected with COVID-19. Firstly, Chengdu formulated and issued the "Working Opinions on the Management of Maternal Women during the Epidemic Period of COVID-19 Infection" immediately after the outbreak. Secondly, some basic information of pregnant women returning from high-risk areas and countries is requested to be reported on a daily basis. Thirdly, a group of experts in Obstetrics, Pediatrics and Hospital Infection Management supervise the implementation of the COVID-19 health care services for pregnant women in primary care institutes. Fourthly, the list of health institutes providing services for confirmed and suspected cases was determined and announced to the public by the government promptly. Additionally, the prevention of mother to infant transmission of HIV is continually strictly practiced during the period of the COVID-19 epidemic. Lastly, all inpatients in the health facilities of Chengdu, including maternity hospitals, are required to have a COVID-19 RT-PCR test to further prevent nosocomial infection. Now, many people living in high-risk countries are coming to Chengdu, and it is therefore necessary to make the prevention and control measures a long-term process.
\end{abstract}

Keywords: COVID-19, pregnant women, control measures, effectively

It has been more than half a year since the first coronavirus disease 2019 (COVID19) case was diagnosed in Wuhan, China. ${ }^{1,2}$ Over 85,000 cases were reported nationally during the period. A public health first-level emergency response was immediately initiated in many provinces. ${ }^{3}$ In January 2020, the Chinese Lunar New Year travel rush led to the rapid spread of COVID-19 across the country. Local government then took serious measures to deal with the outbreak of the novel infectious disease, and afterward the national epidemic has been effectively controlled with the number of new confirmed cases remaining at an incidence level of under 200 per day. ${ }^{4}$ While the COVID-19 outbreak in China was under control, many other countries and areas worldwide are experiencing the COVID-19 pandemic. ${ }^{5}$

Pregnant women are susceptible to infectious diseases including COVID-19. Some early studies have indicated that if pregnant women are infected with respiratory disease, it could have a severe clinical course and lead to adverse 
outcomes. $^{6,7}$ For instance, a pregnant women with COVID-19 experienced miscarriage during the second trimester and it was believed to be related to placental infection with the SARS-CoV-2. ${ }^{8}$ During Chinese New Year, the most important holiday in Chinese culture, people travel long distances to get to their hometowns and to meet their families, which may increase the chance of spreading the severe acute respiratory syndrome coronavirus 2 (SARS-CoV-2) among pregnant women. Therefore, monitoring and management of COVID-19 should be introduced to pregnant women as a part of the prenatal care to minimize the risk of pregnancy complications. ${ }^{9,10}$

Chengdu is the capital city of Sichuan Province with a population of 16 million which had 217 thousand live births in $2019 .{ }^{11}$ It is a typical Chinese city in terms of the level of economic and health system development. A total of 94,770 pregnant women had received health care services in Chengdu by the end of 29 February 2020. As of 10 March 2020, Chengdu managed 406 pregnant women who came from Hubei Province or from abroad, such as South Korea, Japan, Iran, Italy, and who had been in close contact with people from these high-risk areas. So far no suspected cases were detected among pregnant women in Chengdu. In the global pandemic scenario, however, effective implementation of management measures is very important to prevent pregnant women from being infected by the SARS-CoV-2. Sichuan Province enforced the level1 public health emergency response between 24 January and 26 February $2020 .^{12}$ In addition to the general provincial measures, Chengdu dynamically decided to track and manage pregnant women returning from high-risk areas and countries to suppress the spread of COVID-19. There had been no confirmed cases of maternal infection in Chengdu until 24 July 2020, demonstrating that the control and prevention measures were effective.

Chengdu Women's \& Children's Central Hospital is the leading institute providing maternal and child health services in Chengdu. It ecompasses administrative functions of women's health of the whole city, and cooperates with the government departments to perform their duties. Inside the hospital, several precautionary measures are taken to prevent and control COVID-19. The flowchart of management of suspected cases of COVID19 in the hospital is summarized in Figure 1.

Firstly, all people entering the hospital are monitored for body temperature and hand disinfection is mandatory. Guide signs were set at each intersection in the hospital to guide drivers and patients to the fever clinic and emergency department. The pathway of "fever clinic-isolation ward-isolation operating room" was established, together with routes to isolation ward for patients transferred from other hospitals.

Secondly, all patients in our hospital were divided into three levels for pre-examination, which are monitoring of body temperature at "pre-examination inquiries" points of out-patient department (level one), "outpatient inquiries" at nurses' station (level two), and physicians ask the patients about their body temperature (level three)._A medical treatment group was established especially for pregnant women and children infected with COVID-19. All patients, once confirmed to be COVID19 positive, will be treated following the latest version of the Chinese national "Novel coronavirus pneumonia diagnosis and treatment plan". The viral loads detected in the nose were found to be higher than in the throat, ${ }^{13}$ and it is therefore suggested that both nasal swabs and throat swabs should be collected. However, as a large group of patients in our hospital are children, throat swabs were used in most cases due to children's low level of cooperation. Three levels of response were setup in our hospital, and four isolation observation beds are reserved in the emergency department (levelthree response). If there are more than five suspected cases, level-two response will be activated, and if there are more than 15 suspected cases, level-one response will be activated. The human and material resources are prepared and will be allocated accordingly. Specifically, at level-two response, twelve isolation observation beds will be reserved, and nine obstetricians, two neonatologists, six operating room nurses, and six midwives will be allocated to manage the suspected cases. At level-one response, the corresponding number of reserved isolation observation beds, obstetricians, neonatologists, operating room nurses and midwives will be $50,45,10,18$, and 18 , respectively.

Thirdly, special attention is paid to the disinfection of major departments, such as isolation wards, operating rooms, special elevators, and transfer passages. Reuse of surgical instruments in special containers marked as "COVID-19" is handled by the disinfection supply center, and the medical waste is disposed of in accordance with regulations as required by the National Health Committee. ${ }^{14}$ Although there have been no suspected or confirmed cases of COVID-19 in our hospital, strict disinfection measures are taken to prevent nosocomial 


\section{Primary screening of body temperature \\ Level 1: Entrance of outpatient and emergency departments \\ Level 2: outpatient inquries at nurses' station \\ Level 3: physicians ask the patients about their body temperature}

$+$

If with a fever, the patient and his/her escort will be asked to wear a surgical mask, and will be accompanied by a nurse to the fever clinic via a special route

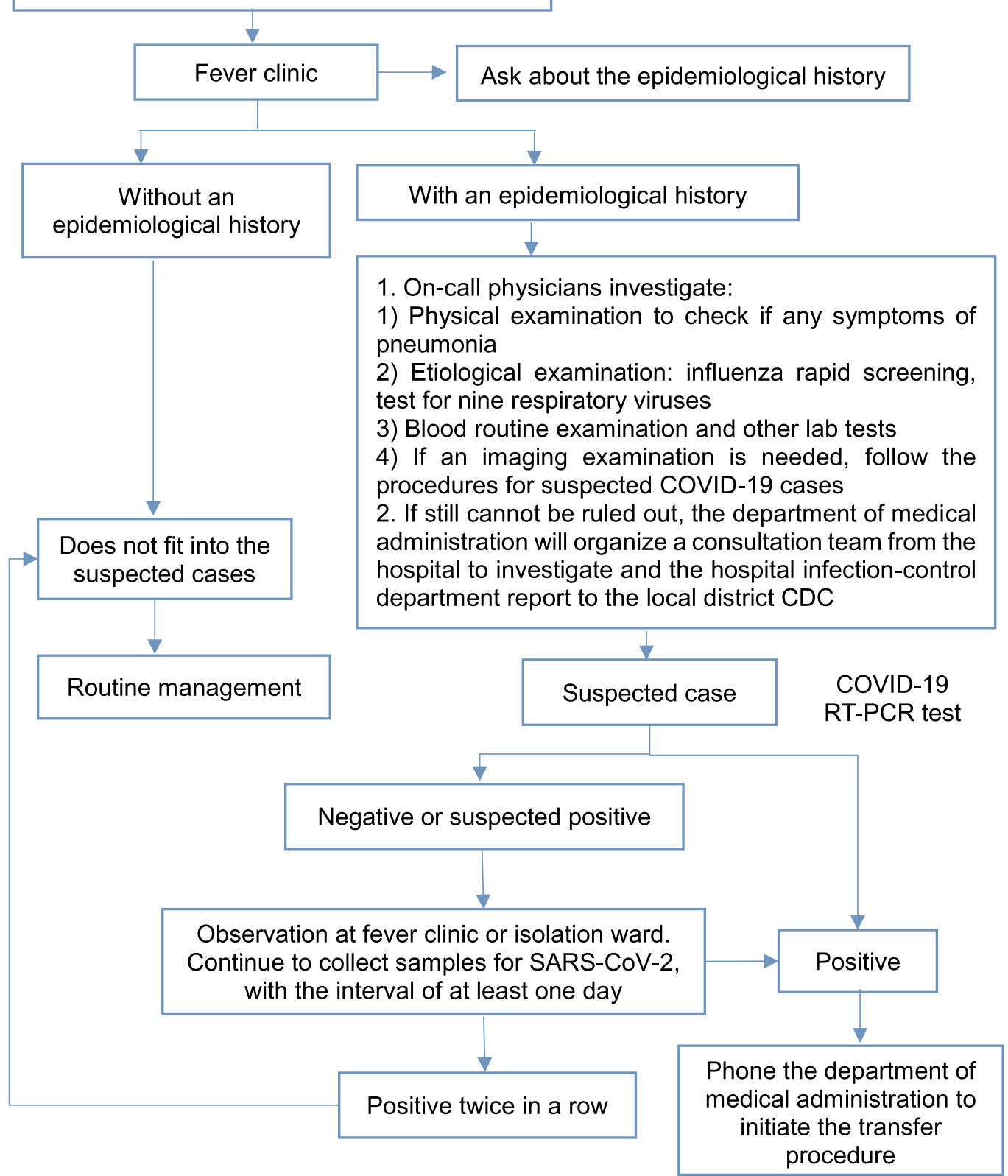

Figure I Flowchart of management of suspected cases of COVID-19 at Chengdu Women's and Children's Central Hospital. 
infection and cross contamination, given that people can spread SARS-CoV-2 before they have symptoms. ${ }^{15}$

\section{Control and Prevention Measures for COVID-I9 Among Pregnant Women in Chengdu}

Firstly, Chengdu formulated the "Working Opinions on the Management of Maternal Women during the Epidemic Period of COVID-19 Infection", which was issued at an early stage of the epidemic. It clarified the direction of maternal health services of the city that all maternity institutes must implement the control and prevention measures according to updated regulations. For instance, postpartum visit should be conducted by telephone, WeChat, online (internet) and other noncontact ways. It is recommended that pregnant women who are in the early stages of pregnancy and without special conditions should extend the visit intervals of scheduled health care in the hospital. However, pregnant women in the middle and late stages of pregnancy and those with high-risk factors still need to go to hospital for health care regularly.

Secondly, immediate submission of basic information related to COVID-19 among pregnant women returning from high-risk areas and countries is requested to be reported every day. Starting from 26 January 2020, the information of pregnant women (including basic information, expected delivery date, high-risk situations and medical isolation period) who have come from areas such as Hubei, Chongqing, Japan, and Korea, who have had close contact with suspected or confirmed cases, are suspected or confirmed cases, is collected and submitted promptly by the primary health care workers, so as to provide an information basis for the city to fight COVID-19. Each pregnant woman who had close contact with those suspected/confirmed cases was tracked down and managed. They need to self-isolate themselves at home. The primary health care workers contact these pregnant women once a day by telephone to find out their body temperature. Once any suspicious symptoms are present (such as fever, cough, and difficulty breathing), pregnant women are required to report their status to the primary health care workers by telephone as soon as possible.

Thirdly, supervising of the implementation of the COVID-19 health care services for pregnant women in primary care institutes had been carried out. As of 23 January 2020, experts in Obstetrics, Pediatrics and Hospital Infection Management are organized in groups to cover all districts and counties of the city. Experts and public officials mainly visited maternal and child health care institutions and private medical institutions to supervise the implementation of pre-examination, fever clinic, isolation waiting point and isolation ward. At the same time, experts also gave guidance regarding the management of postpartum women and newborns during the isolation period.

Fourthly, government urgently investigated the service capacity of the city's two-level critical care centers and maternal and child health hospitals for COVID-19 (including negative pressure ward, negative pressure delivery room, and negative pressure operating room), and determined the list of institutions for admission and treatment of confirmed and suspected cases distributed in 22 districts and counties throughout the city, and announced it to the public. At the same time, the municipal Emergency Delivery Office promptly forwarded the "Letter of information on the close contact of XX from the maternal and child health care institutions" for reference of midwifery institutions.

Fifthly, government strengthened the monitoring and management of the special groups (HIV infected women of childbearing age, pregnant women and their children). The files on prevention of mother to child transmission during the period of COVID-19 were detailed, implemented and issued immediately. In addition, a special obstetric emergency drill about COVID-19 infection was carried out in a maternal and child health care hospital, which helped obstetricians to observe and make themselves "master of methods" for dealing with suspected and infected COVID19 pregnant women.

Lastly, as required by Chengdu Municipal Health Commission, all inpatients in the health facilities of Chengdu, including maternity hospitals, should have a COVID-19 RT-PCR test to identify asymptomatic or minimally symptomatic individuals and to help further prevent nosocomial infection.

\section{Challenges and Suggestions}

Chengdu made significant efforts and got some achievements in controlling the COVID-19 epidemic among pregnant women. Despite the fact that there is no report of COVID-19 infection among pregnant women in Chengdu, World Health Organization has announced the COVID-19 outbreak a global pandemic. Every day, many people residing in other countries are coming to Chengdu and pregnant women may have close contact with these people. Facing rising pressure of guarding against imported cases and preventing a resurgence of the outbreak at home, 
strict prevention and control measures should not be neglected. As the cases of COVID-19 have reached alarming levels in many countries, it is necessary to make prevention and control interventions a regular and longterm process. What we have learned from the COVID-19 epidemic and pandemic may help to improve the effectiveness of future clinical practices in terms of diagnosis and treatment of new infectious diseases. However, more consideration should be given to the allocation of medical human resources, particularly in scenarios where doctors and nurses are infected with COVID-19.

\section{Disclosure}

The authors report no conflicts of interest for this work.

\section{References}

1. Huang C, Wang Y, Li X, et al. Clinical features of patients infected with 2019 novel coronavirus in Wuhan, China. Lancet. 2020;395 (10223):497-506. doi:10.1016/S0140-6736(20)30183-5

2. Zhu N, Zhang D, Wang W, et al. A Novel Coronavirus from Patients with Pneumonia in China, 2019. $N$ Eng J Med. 2020;382(8):727-733. doi:10.1056/NEJMoa2001017

3. The People's Government of Sichuan Province. [Notice of Novel Coronavirus Infection I Response Measures Issued by the General Office of Sichuan Provincial People's Government]. Chengdu; 2020. Chinese.

4. Health Emergency Office. [Updates on COVID-19 as of 28 July 2020]. Available from: http://www.nhc.gov.cn/yjb/s7860/202007/ 367ebba964864a768d4239f13774e1e4.shtml. Accessed 29 July 2020.

5. WHO. WHO Characterizes COVID-19 as a Pandemic; 2020.
6. Rasmussen SA, Smulian JC, Lednicky JA, Wen TS, Jamieson DJ. Coronavirus Disease 2019 (COVID-19) and pregnancy: what obstetricians need to know. Am J Obstet Gynecol. 2020;2:214.

7. Chen H, Guo J, Wang C, et al. Clinical characteristics and intrauterine vertical transmission potential of COVID-19 infection in nine pregnant women: a retrospective review of medical records. Lancet. 2020;395(10226):809-815. doi:10.1016/S0140-6736(20)30360-3

8. Baud D, Greub G, Favre G, et al. Second-Trimester Miscarriage in a Pregnant Woman With SARS-CoV-2 Infection. JAMA. 2020;323 (21):2198-2200. doi:10.1001/jama.2020.7233

9. Qiao J. What are the risks of COVID-19 infection in pregnant women? Lancet. 2020;395(10226):760-762. doi:10.1016/S01406736(20)30365-2

10. National Health Commission of the People's Republic of China. [Notice on Strengthening Maternal Disease Treatment and Safe Midwifery During the Prevention and Control of New Coronavirus Pneumonia]; 2020. Chinese.

11. The People's Government of Sichuan Province. Press Conference of Sichuan Provincial Demographic Bulletin in 2018; 2019.

12. ChinaNews. [Sichuan activates level-I public health emergency response]. 2020. Available from: https://m.chinanews.com/wap/ detail/zw/gn/2020/01-24/9068977.shtml. Accessed October 11, 2020. Chinese.

13. Zou L, Ruan F, Huang M, et al. SARS-CoV-2 Viral Load in Upper Respiratory Specimens of Infected Patients. N Engl J Med. 2020;382 (12):1177-1179. doi:10.1056/NEJMc2001737

14. General Office of National Health Commission of the People's Republic of China. [Circular of the General Office of National Health Commission on the Management of Medical Waste in Medical Institutions During the Epidemic Period of COVID-19]. Beijing; 2020. Chinese.

15. Long Q-X, Tang X-J, Shi Q-L, et al. Clinical and immunological assessment of asymptomatic SARS-CoV-2 infections. Nat Med. 2020;26(8):1200-1204. doi:10.1038/s41591-020-0965-6
Risk Management and Healthcare Policy

\section{Publish your work in this journal}

Risk Management and Healthcare Policy is an international, peerreviewed, open access journal focusing on all aspects of public health, policy, and preventative measures to promote good health and improve morbidity and mortality in the population. The journal welcomes submitted papers covering original research, basic science, clinical \& epidemiological studies, reviews and evaluations, guidelines, expert opinion and commentary, case reports and extended reports. The manuscript management system is completely online and includes a very quick and fair peer-review system, which is all easy to use. Visit http://www.dovepress.com/testimonials.php to read real quotes from published authors. 